\title{
Electric Vehicle Charging to Support Renewable Energy Integration in a Capacity Constrained Electricity Grid
}

\author{
Nathaniel S. Pearre \\ Lukas G. Swan \\ Renewable Energy Storage Laboratory, Dept. of Mechanical Engineering, Dalhousie University, PO Box \\ 15000, Halifax, Nova Scotia, B3H 4R2, Canada \\ Corresponding Author: \\ Nathaniel S. Pearre \\ natpearre@gmail.com \\ (902) 403-8583 \\ Keywords: Smart Grid; Renewable energy; Transmission constraint; Electric vehicle; Driving survey
}




\section{Abstract}

21 Digby, Nova Scotia, is a largely rural area with a wealth of renewable energy resources, principally

22 wind and tidal. Digby's electrical load is serviced by an aging $69 \mathrm{kV}$ transmission line that often

23 operates at the export capacity limit because of a local wind energy converter (WEC) field. This

24 study examines the potential of smart charging of electric vehicles (EV) to achieve two objectives:

25 1) add load so as to increase export capacity; 2) charge EVs using renewable energy.

26 Multiple survey instruments were used to determine transportation energy needs and travel

27 timing. These were used to create EV charging load timeseries based on "convenience", "time-of-

28 day", and idealized "smart" charging. These charging scenarios were evaluated in combination with

29 high resolution data of generation at the wind field, electrical flow through the transmission 30 system, and electricity load.

31 With a $10 \%$ adoption rate of EVs, time-of-day charging increased local renewable energy usage by $32 \quad 20 \%$ and enables marginal WEC upgrading. Smart charging increases charging by local renewable 33 energy by 73\%. More significantly, it adds $3 \mathrm{MW}$ of load when power exports face constraints, 34 allowing enough additional renewable electricity generation capacity to fully power those vehicles. 


\section{Table of contents}

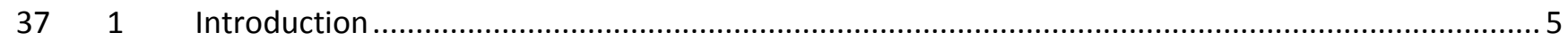

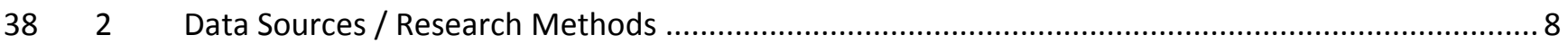

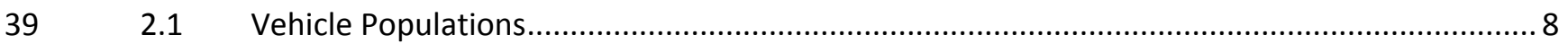

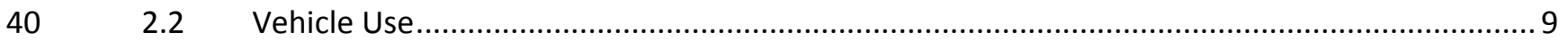

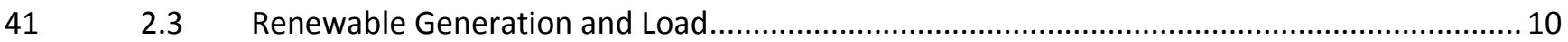

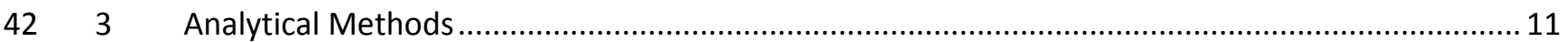

$43 \quad 3.1 \quad$ Annual electricity consumption of a Digby electric vehicle population ................................ 11

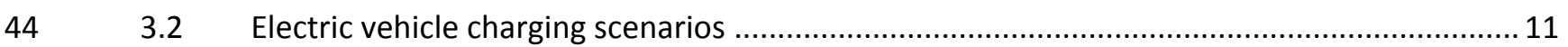

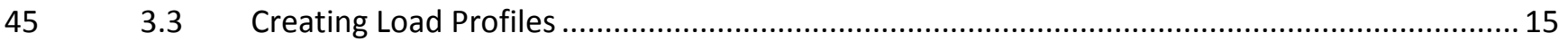

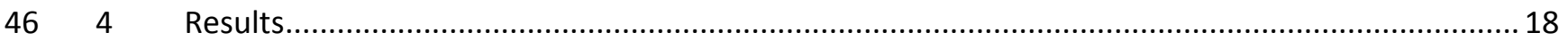

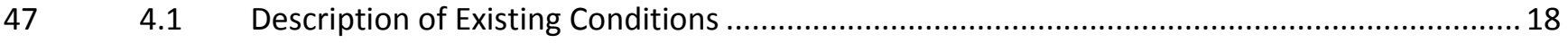

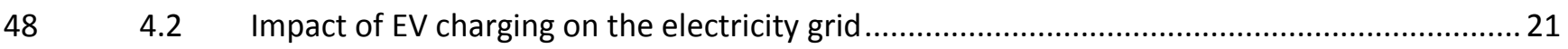

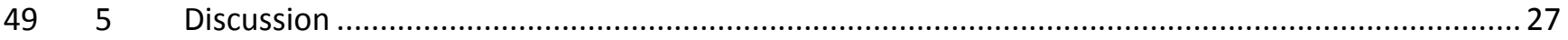

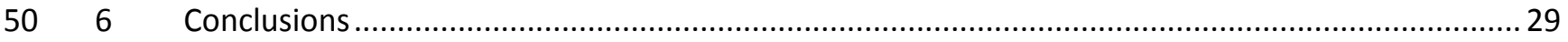

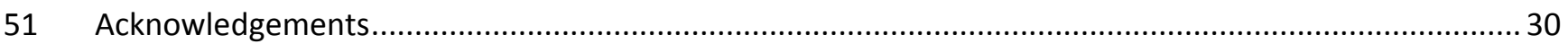




\section{Nomenclature}

\begin{tabular}{|l|l|}
\hline EV & Electric vehicle \\
\hline TOD & Time Of Day; electric rates that vary $(\$ / \mathrm{kWh})$ on a fixed schedule \\
\hline WEC & Wind Energy Converter (wind turbine) \\
\hline
\end{tabular}

53 


\section{Introduction}

55 The Municipality of Digby (Figure 1, left) is embarking on an ambitious strategy to alter its energy

56 consumption and production, for greater utilization of locally produced renewable energy.

57 Transportation represents a major energy end-user, totalling 38\% of all energy used in Nova Scotia

58 [1]. This energy comes almost entirely in the form of gasoline and diesel. While Canada has

59 significant fossil fuel resources, there is no petroleum production in Nova Scotia, so transportation

60 fuels represent a significant economic trade deficit for the region. In contrast, Nova Scotia in

61 general, and the Digby area in particular, have superb renewable energy resources consisting

62 principally of wind and tidal flows $[2,3]$. Electric vehicles (EVs) which have greatly increased

63 efficiency compared with internal combustion engines, thus represent an opportunity to not only

64 vastly reduce energy consumption for transportation, but also to transition from imported fossil

65 fuels to locally produced renewable energy.

66

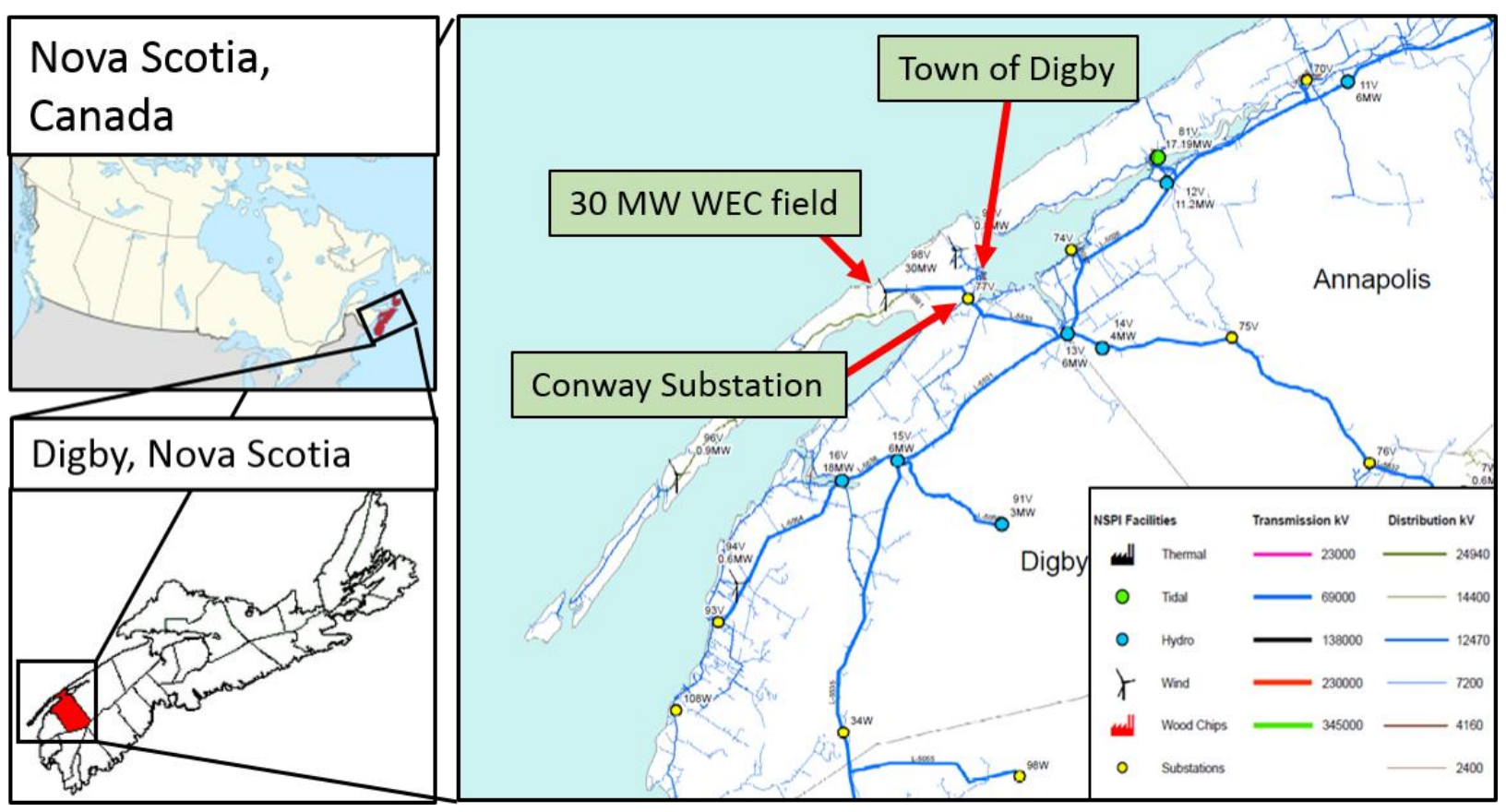

67

Figure 1 Location of Digby (left) and transmission and distribution maps (right) 
The electrical transmission system of the area is shown in Figure 1 (right). It consists of $69 \mathrm{kV}$ lines servicing the Town of Digby via Conway Substation. Other $69 \mathrm{kV}$ lines connect nearby communities and collect from small hydroelectric facilities inland. In 2010 a $30 \mathrm{MW}$ wind energy converter (WEC) field, consisting of twenty GE 1.5 MW units, was commissioned on the Digby Neck, causing Digby to become a net exporter of electricity. This $30 \mathrm{MW}$ wind field was sized to meet summertime transmission export limits when local loads are at their minimum. As a consequence, further development of renewable electricity generation is not permitted, absent one of three conditions: Either 1) the transmission system is upgraded to increase the export capacity, 2) renewable generation must be curtailed when transmission limits are reached, or 3) Electrical load must be added locally, so that the additional power produced can be used locally and not contribute to overloading of the transmission system.

Option 1 is not being considered by the provincial electricity system manager in short or long term planning because it would be prohibitively expensive. Option 2, while not presently supported by the grid manager, is a reactive approach that is undesirable due to the loss in renewable energy caused by curtailment. It is under the premise of Option 3 that this study is conducted. The addition of EVs adds load to the local electricity network. By evaluating the time-dependent load of charging EVs and their interaction with existing loads and generation, this study will quantify the influence of EVs on the electricity grid for a local region, and the use of renewable electricity generation for powering those EVs.

The use of energy storage and dispatchable load to manage variations in renewable energy output is a problem of nearly universal concern in utility management as non-dispatchable renewable energy sources become a significant contributor to total energy and grid instability [4-7]. Applying the possible grid management benefits of EV charging to achieve a specific grid management objective is of great interest to governments and utilities $[8,9]$, so this case study, which benefits 
92 from well-defined grid constraints and precise grid loading data, may be of particular interest to 93 policy makers.

94 The interaction of EVs that plug into the electrical system, and the electrical system itself rely 95 entirely on an accurate understanding of when EVs are used, how much energy they consume when 96 they are used, and when they are returned to a location where they can plug in and charge. The 97 significance of the driving patterns is made doubly important when one considers three possible 98 effects of EVs on the energy system [10]. One possibility is an undesirable evening peak in load that 99 could occur if charging rates and timing are unconstrained, referred to here as "convenience 100 charging". The second is to respond to "time of day" (TOD) electricity rates with a charge timer, in 101 which case an evening load peak is avoided and loads overnight are increased, but no more finely 102 tailored benefits can be realized $[11,12]$. The third possibility is "smart charging", which is 103 managed by grid operator intelligence and real-time control, in which EV charging loads become a 104 controllable resource providing valuable grid services.

105 Any charging strategies that are successful in reducing or controlling the export transmission loads 106 could correspondingly permit increased local generation capacity. General Electric (GE), the 107 manufacturer of the WECs in use at the wind field have developed a control software update titled 108 WindBOOST, which increases the maximum power output by 10\%, from $1.5 \mathrm{MW}$ to $1.65 \mathrm{MW}$. This 109 modification could be implemented at negligible cost, and would increase WEC field power 110 capability to $33 \mathrm{MW}$, and annual average energy production by roughly $4 \%[13,14]$. As an objective, 111 this study investigates the potential of adding controlled EV charging, thus allowing the 112 WindBOOST upgrade, with the intent that the added energy production would be sufficient to 113 provide the necessary energy to charge the EVs, making them a net benefit to Nova Scotia's grid. 


\section{Data Sources / Research Methods}

115 To conduct a thorough investigation of EVs and their impacts upon the electrical grid requires an 116 understanding of the present transportation fleet in Digby with respect to both vehicle populations 117 and vehicle use. Specifically, to understand the energy requirements of vehicles, it is necessary to 118 know 1) how many vehicles of various types are in use in the area, 2) how much energy these 119 vehicles use each day (how far they drive and how much fuel is consumed to do so), and 3) during 120 what period of the day, and particularly when at the end of the working day, they are parked, 121 indicating when vehicles would plug in to the electricity grid. With those data and an assumed 122 adoption rate of EVs, grid impacts can be estimated.

123 The following subsections describe the regions of analysis, the data sources related to vehicle 124 populations in the area, the survey tools used to gather vehicle use information, and the data 125 available on grid loading and renewable electricity generation.

126

127 128 129

\subsection{Vehicle Populations}

The total vehicle population in Digby comes from Provincial vehicle registration data [15], however, the population served by the Conway electrical substation (Figure 1) does not correspond to a specific Provincial jurisdiction. The inferred population of vehicles must therefore be computed by scaling data from:

- $\quad$ The Town of Digby with an area of approximately $3 \mathrm{~km}^{2}$ and a population of 2152 .

- Digby County, consisting of the Town (above) and two Municipal Districts which combined have an area of approximately $2,515 \mathrm{~km}^{2}$ and a population of 18,036.

To establish the population served by Conway, estimates of Town and Municipal population were combined with a building count using satellite imagery. The resulting population estimate for the Conway service area is 9000 people. Table 1 groups the transportation fleet of Digby by vehicle type, lists how many of each type are registered in the Town and in the County, and gives the 
140 this population estimate.

Table 1 Classes of vehicles, estimated numbers that operate in the regions of analysis in Digby

\begin{tabular}{l|rcc|}
\hline Vehicle type & $\begin{array}{c}\text { Digby Town } \\
\text { (count) }\end{array}$ & $\begin{array}{c}\text { Conway Area } \\
\text { (estimate) }\end{array}$ & $\begin{array}{c}\text { Digby County } \\
\text { (count) }\end{array}$ \\
\hline Population & 2152 & 9000 & 18,036 \\
\hline Motorcycle & 74 & 316 & 646 \\
\hline Small Car & 353 & 1275 & 2151 \\
\hline Medium Car & 347 & 1238 & 2053 \\
\hline Large Car & 247 & 884 & 1472 \\
\hline Van / SUV & 131 & 504 & 921 \\
\hline Pickup & 370 & 1577 & 3218 \\
\hline ATV & 76 & 401 & 971 \\
\hline Bus (diesel) & 0 & 9 & 38 \\
\hline School Bus (diesel) & 0 & 3 & 13 \\
\hline Freight Van (diesel) & 105 & 356 & 546 \\
\hline
\end{tabular}

142

143

\subsection{Vehicle Use}

To gather information on vehicle use, energy consumption, and the timing when EVs would be plugged-in, a variety of survey techniques were used.

To gather information on business vehicles, a selection of businesses in Digby County were interviewed by telephone. The selection of business types called for this study was made based on the perceived likelihood that they would have commercial vehicles (i.e. registered vehicles used exclusively or primarily by the business). Forty-seven business were interviewed by phone. Respondents were asked what business vehicles they used, how much they were used, and when during the day such use took place. Where specific information was not provided, average values for the vehicle type were used.

To gather the corresponding information about Digby area household vehicle use, both telephone and online survey methods were used. A total of 22 households were interviewed in depth in 
155 telephone surveys. In addition, there were 79 unique responses to an online survey that was

156 promoted on the Municipality's web-site, and a newspaper advertisement.

\section{$157 \quad 2.3$ Renewable Generation and Load}

158 A variety of data concerning local, regional, and provincial electrical system conditions, including

159 WEC field production, were available for this research, supplied by the provincial utility, Nova

160 Scotia Power Inc. or its affiliates. The variables available to this research are shown in Table 2

161 (refer also to Figure 1, right, for a map of electrical grid infrastructure). Note that for most of the

162 system loading analysis, power transmitted on L5581 is used for the WEC field output, rather than

163 the turbine data referred to in the first line of Table 2.

164 Table 2 Digby electricity infrastructure measured data points and sampling rates

\begin{tabular}{|c|c|c|c|c|c|}
\hline Category & Name & Detail & Variables & $\begin{array}{l}\text { Sampling } \\
\text { rate }\end{array}$ & $\begin{array}{l}\text { Data } \\
\text { collection } \\
\text { period } \\
\end{array}$ \\
\hline Generation & WEC field & 30 MW capacity & $\begin{array}{l}\text { Wind speed, } \\
\text { Wind direction, } \\
\text { Ambient } \\
\text { temperature, } \\
\text { Real power, etc. }\end{array}$ & 10 minute & $\begin{array}{l}\text { Jan } 2011- \\
\text { Dec } 2013\end{array}$ \\
\hline \multirow{2}{*}{ Transmission } & Line L-5581 & $\begin{array}{l}\text { Connects WEC field } \\
\text { to Conway }\end{array}$ & Real power, & \multirow{3}{*}{20 second } & \multirow{4}{*}{$\begin{array}{l}\text { Jan } 2014- \\
\text { Jun } 2014\end{array}$} \\
\hline & Line L-5533 & $\begin{array}{l}\text { Connects Conway } \\
\text { to Provincial Grid }\end{array}$ & Reactive power & & \\
\hline Distribution & $\begin{array}{l}\text { Distribution } \\
\text { Lines } 301 \text { 302, } \\
303\end{array}$ & $\begin{array}{l}\text { Connects Conway } \\
\text { to Digby Loads }\end{array}$ & $\begin{array}{l}\text { 3-phase } \\
\text { currents }\end{array}$ & & \\
\hline Substation & Conway (77V) & Transformer & Primary voltage & 1 minute & \\
\hline
\end{tabular}




\section{Analytical Methods}

167 In this section, the steps taken to transform the survey and energy system data into an impact 168 analysis are described.

169

\subsection{Annual electricity consumption of a Digby electric vehicle population}

In order to determine the cumulative impacts of EVs in Digby, we assume an adoption rate corresponding uniformly to all vehicle classes of 10\% (approximately 600 count). Although aggressive, it is attainable over the medium term given both local and provincial transportation electrification policies [16]. Regardless of technological advances and economies of scale of EVs to support consumer purchase, an adoption rate of $10 \%$ takes significant time because of the role-over time of the existing fleet.

These adoption rates do not take into account the differences in behaviors of commercial and private vehicle owners. Commercial vehicle owners may have the financial tools to amortize a high upfront cost and recoup it through operational savings, where private vehicle owners may not; however, private vehicle owners presently have a more comprehensive market and more local dealer engagement. Estimating how these aspects will play off against each other in the coming years is a challenge, so we have defaulted to the equal adoption assumption.

\subsection{Electric vehicle charging scenarios}

There are three scenarios of EV charging control that represent a range of technology and vehicle grid interactivity. In this section they are described in the context of how they might operate in the Digby area.

\section{Scenario: Convenience charging}

The "convenience charging" case is conceptually similar to mobile phones, in that the vehicle is plugged in and charged right away upon reaching a charge station, without regard to the time of 
day or the effect on the grid. In this scenario, EVs are likely plugged in immediately upon arriving at 190 a destination, typically home, and are charged until they are full. The control logic for this charging 191 scenario is detailed in Figure 2. The effect of this charging behavior would not be very different in 192 Digby than in other regions where this scenario results in a charging load peak between $17 \mathrm{~h}$ and $19319 \mathrm{~h}[17,18]$, unless there are systematic differences in the driving patterns of vehicles. The 194 majority of EVs presently in Nova Scotia charge using convenience charging, because no provincial 195 or utility policy exists to motivate any other behavior.

\section{For Each Time Increment, For Each Car}

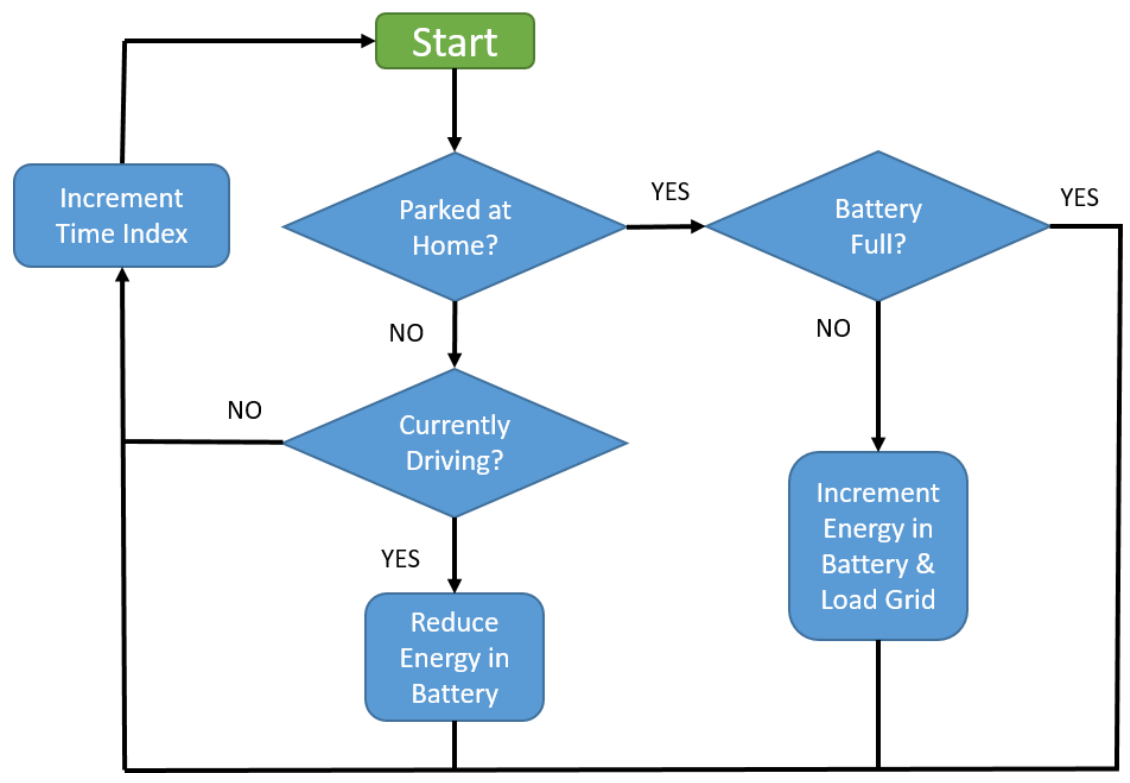

Figure 2 Logic Diagram for "Simple Charging" algorithm. The Output is a load profile of each vehicle, which are then aggregated to become fleet charging loads.

\section{Scenario: Time of day charging}

200 Nova Scotia has in place a "time of day" (TOD) residential electricity tariff, presently available to

201 households with electric-thermal-storage. Three different rates are applied to electrical energy

202 consumed at different times of the day, week, and year. The three rates represent a significant variation in price, especially during the winter peaking months of December through February, as illustrated in Figure 3 [19]. 


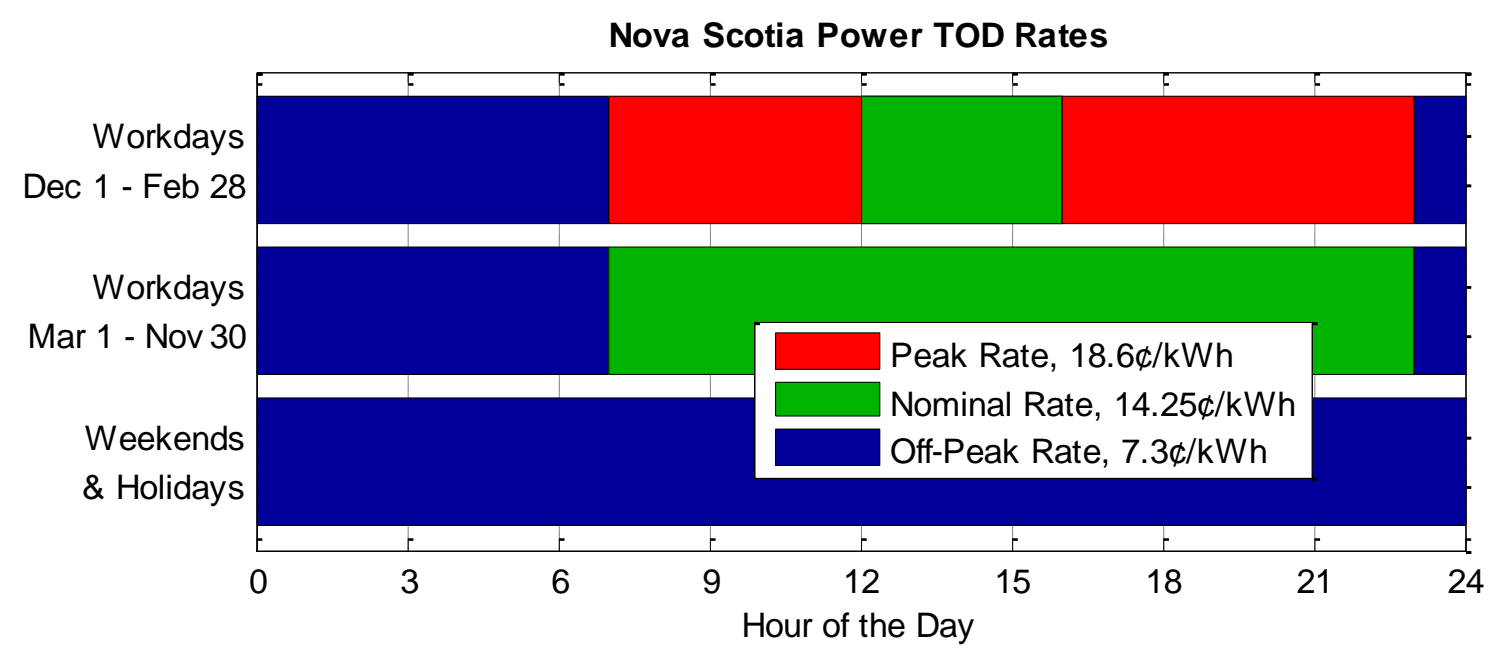

Figure 3 Nova Scotia Power domestic service time-of-day electricity tariff schedule

207 While the rate structure shown in Figure 3 is somewhat complex, for the purposes of this analysis

208 the assumed response by EV drivers to this TOD charging scenario will be to delay charging 209 through the use of a charge timer until after 23h if possible, regardless of the day of the week, or the 210 season of the year. While a more complex response is possible, it is unlikely that charge timing 211 would differentiate between workdays and weekends, and so is uniformly applied for this study.

212 The control logic for this charging scenario is detailed in Figure 4. It is almost identical in structure 213 to that used for simple charging, with the added test to insure charging does not take place during 214 the day. 
For Each Time Increment, For Each Car

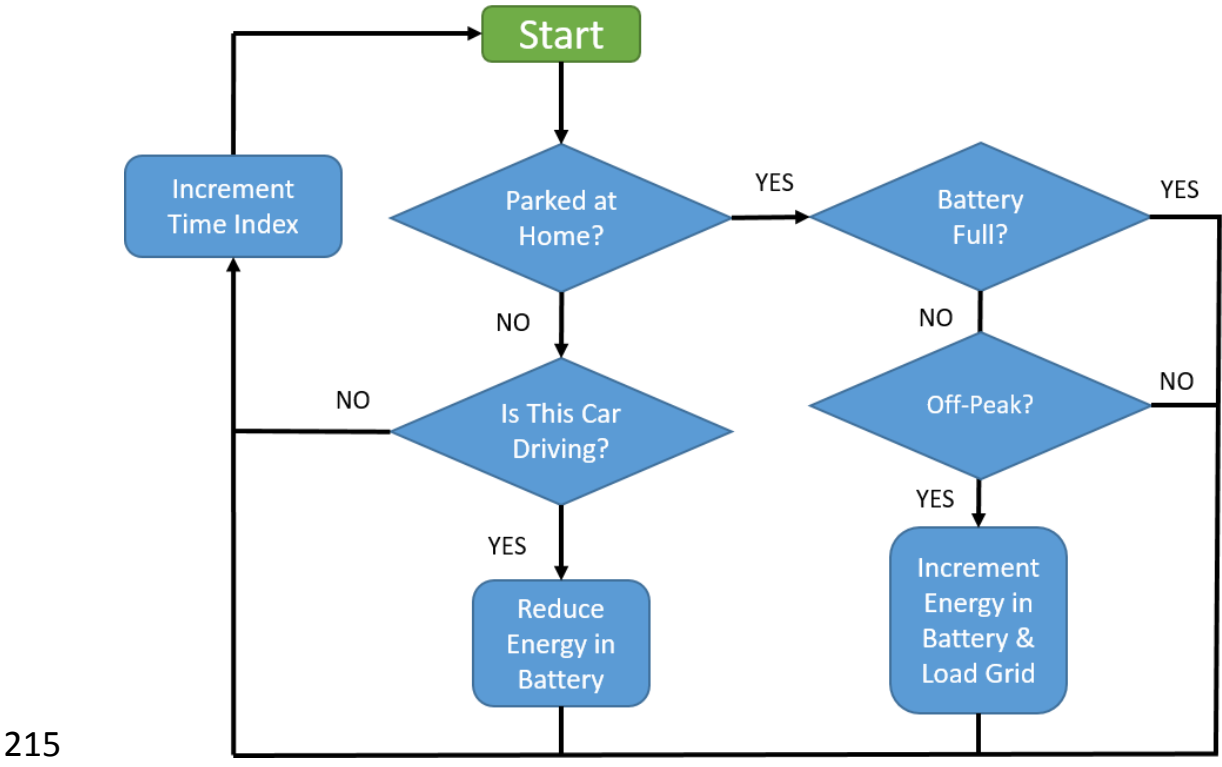

216

217

Figure 4 Logic Diagram for "TOD Charging" algorithm. Output is load profile of each vehicle, which are then aggregated to become fleet charging loads.

\section{Scenario: Smart charging}

In a "smart charging" scenario, the EV is responsive to conditions and constraints which exist on the electricity grid or in electricity markets. In Digby, the constraint of interest is power export on transmission line L-5533 (see Figure 1). When the WEC field is at maximum generating capacity (30 MW), and the Digby load is low, the transmission line reaches its export limitation. With real-time monitoring, coupled with signaling, the net export on this transmission line could be used to signal EVs the preferred time to charge, and how fast to charge. When signalled, all the EVs with any flexible or discretionary charging capacity (i.e., those vehicles that are plugged in but not already charging) could begin charging, thereby increasing the Digby load and decreasing the net electricity export.

To model the effects of such a strategy, each 24 hour period (noon to noon, so overnight discontinuities are avoided) was examined in isolation, and charging loads were added to periods when export power was at its highest for the period. The control logic for the smart charging 
231 scenario is detailed in Figure 5. The logical structure of this scenario is significantly different from

232 those of the previous two (Figure 2 and Figure 4), most notably because the unit of analysis is the 23324 hour period, rather than each vehicle. It should be noted that in the model as configured, a 234 perfect response to this signal was used. In reality, any charging signal based on either a fixed 235 schedule (TOD), or based on some real-time external signal, would only be a suggestion or a price 236 difference, not a strict command. By default, when the vehicle requires charging for an imminent 237 trip, charging will take place regardless of the grid impacts.

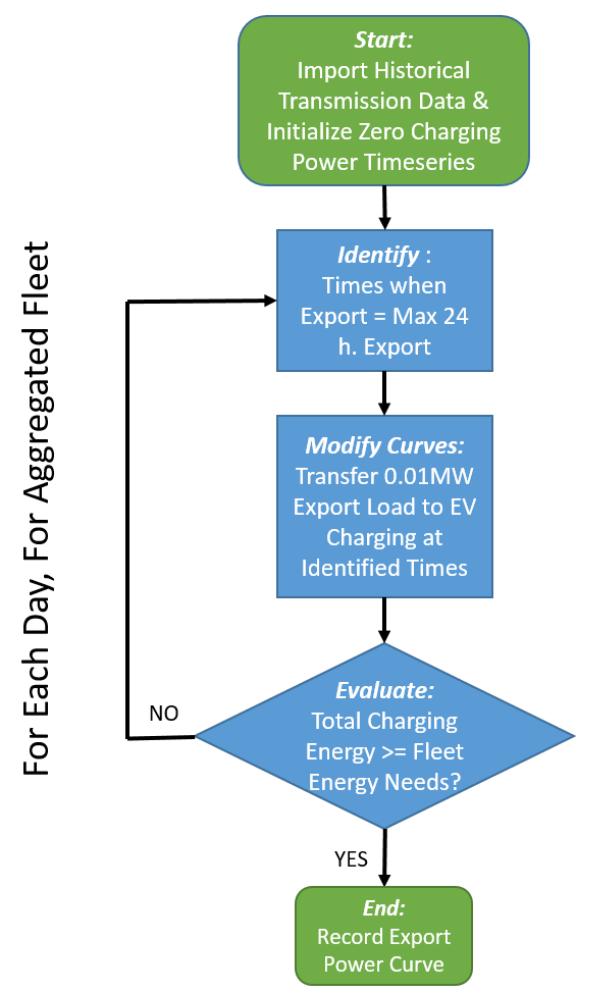

Figure 5 Logic Diagram for "Smart Charging" algorithm. Output is load profile of each 24 hour period, which are then compiled in sequence to become fleet charging loads.

\subsection{Creating Load Profiles}

242 Based on the vehicle counts in Table 1 and coverage of the Conway Substation in Figure 1, we assume 6000 vehicles exist in the area, and that the $10 \%$ adoption rate gives 600 EVs. Based on the survey of driving patterns, and vehicle class specific energy needs, Digby's EVs would need an average of $15 \mathrm{kWh}$ per day, representing a fleet average of passenger and commercial vehicles. 
246 While this value is $2-3$ times the daily energy needs assumed by some previous studies $[9,20]$, this

247 difference can be largely attributed to the assumption made here of proportional EV adoption

248 across all vehicle classes, while those studies focused on small and mid-sized passenger cars.

249 The outputs of the vehicle use surveys were a set of individual vehicle use patterns and average 250 daily energy needs on generic weekdays and generic weekend days. For each vehicle, these outputs 251 were used to construct individual charging load profiles for convenience charging by drawing 252 electrical load right away when returned to a home parking location, or for TOD charging by 253 drawing load at $23 \mathrm{~h}$ if parked at home (refer to section 3.2).

254 For the class of private vehicles and the class of business EVs, the average loads of all survey 255 respondents' vehicles were proportioned equally to that of the total population of privately owned 256 and commercial vehicles, respectively. Commercial and private charging load profiles were then 257 multiplied by the presumed adoption fraction for each class ( $10 \%$ for both), and summed together 258 to produce a regional vehicle fleet charging load for each day class, for each of the first two charging 259 scenarios.

260 To produce a smart charging load profile, the sum of vehicle energy was used to 'fill' the points of 261 greatest export power in each noon to noon 'day'. For example, at a historical export power peak of $262-25 \mathrm{MW}$, a modified peak export power of -24.99 MW was specified, and the difference in energy 263 between an export power timeseries bottoming out on the modified peak and one following the 264 historical data was computed. If that summed energy was less than the necessary driving energy for 265 the fleet, then the modified peak export power was increased again to -24.98 MW. This process was 266 iterated until the difference between the original and 'capped' export energy curves was equal to 267 the energy needs of the EV fleet. This process is illustrated as a flow diagram in Figure 5. 
268 This methodology for smart charging could, in theory, require charging at any time, and possibly 269 when a significant fraction of the fleet was not parked. However, this does not adversely affect the 270 analytical results, because problematic peaks in power export can only occur when both wind 271 production is high and when local loads are low, and the later condition occurs only at night. Thus 272 the test points for the system, the times at which the transmission system nears capacity limit, can 273 only be at night when most vehicles are parked. 


\section{Results}

275 In this section, the interactions and influence of EVs on the electric grid are discussed, with a focus

276 on how they would relate to renewable electricity generation in Digby.

\subsection{Description of Existing Conditions}

278 Figure 6 shows monthly average, and maximum and minimum electricity load in Digby, and generation at the WEC field. These data are from 2014, and 2012 through 2013 respectively, and it can be noted that only six months of data are available for Digby as the substation monitoring equipment was recently installed. These various years are not expected to affect the trending

282 shown in Figure 6.
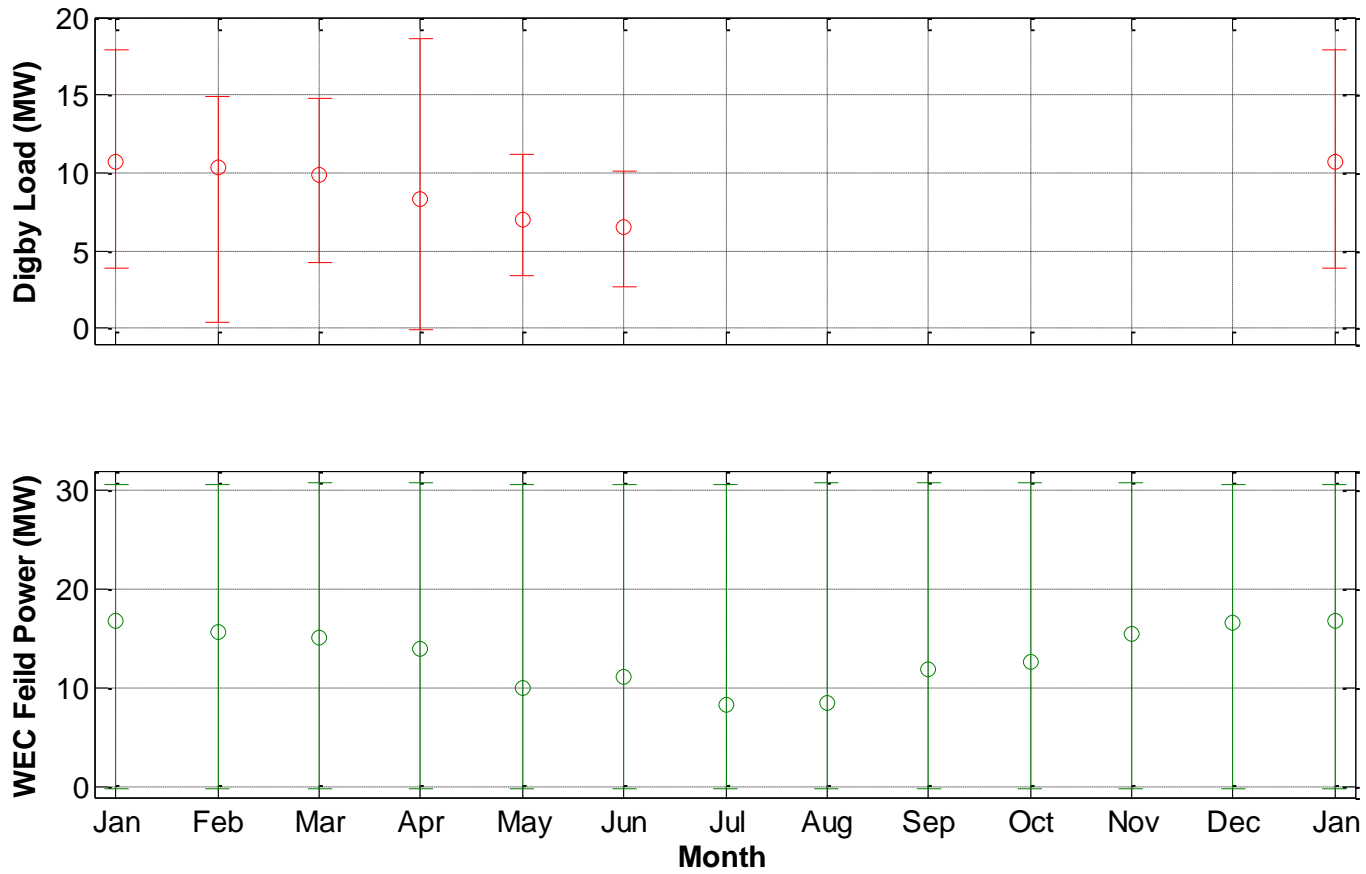
as range bars for Digby load (top, red), and $30 \mathrm{MW}$ WEC field generation (bottom, green).

In general, load in winter is higher than the load in summer, due to increased need for space- and

287 water- heating, and increased need for artificial light as daylight hours recede. Similarly, winds are 
stronger in winter, and thus WEC field generated electricity follows a similar seasonal pattern. The

289 WEC field generation averages 13 MW, while Digby averages roughly 8.8 MW of load, meaning the 290 Digby region is an annual net exporter of electricity. Also shown on Figure 6 are monthly maximum 291 and minimum values as range bars about the averages. It can be seen that Digby's load can reach 292 well above $18 \mathrm{MW}$, though only briefly, and can also drop to zero (during a power outage). The WEC 293 field's output is continuously variable, achieving slightly more than its rated capacity of $30 \mathrm{MW}$ and 294 also becoming a small net load (<0 MW) within every month.

295 Of greater interest for EVs charging and grid management, Figure 7 shows the hourly average load 296 for each of six pairs of months, and shows how load and wind generation vary throughout the day. 297 The different colored lines show different daily profiles characteristic of different seasons. From 298 Figure 7, the load in Digby (top), in both cold and warm months can be seen to drop by $2-3$ MW 299 overnight, with a minimum occurring between $2 \mathrm{~h}$ and $4 \mathrm{~h}$. During the winter months, loads peaks 300 twice daily, in the late morning and again in evening. This is characteristic of areas with electric 301 heat that is often turned down at night when businesses are empty and people are asleep, and in 302 homes during the day when people are at work. ${ }^{1}$

\footnotetext{
${ }^{1}$ A comparison to Provincial load data suggest that Jul-Aug and Sep-Oct load curves would likely be similar to those of May-Jun, while the load curve for Nov-Dec would lie between those of Jan-Feb and Mar-Apr.
} 


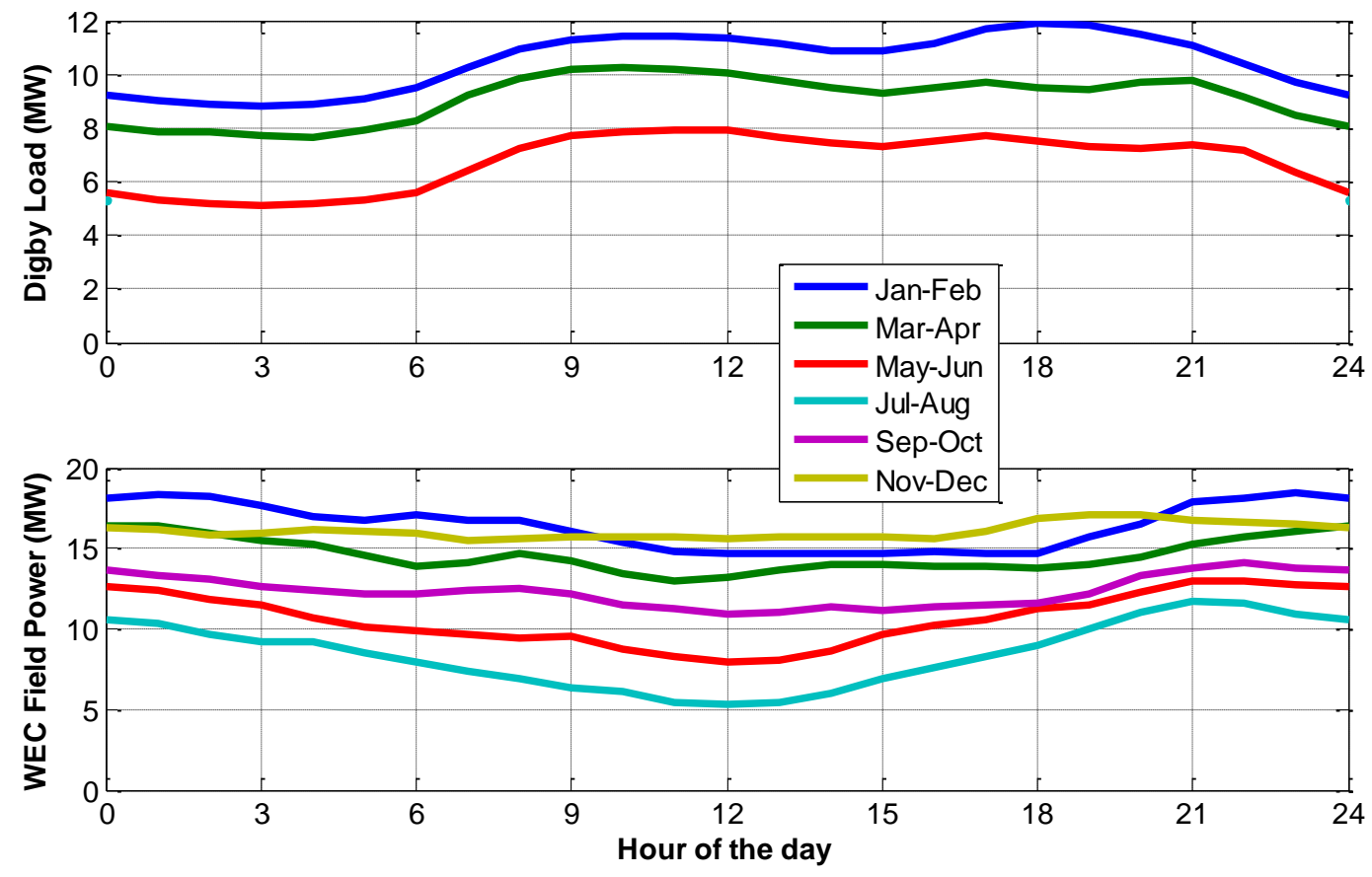

Figure 7: Average electricity power as a function of month pairs (colored) for Digby load (top), and 30 MW WEC field generation (bottom)

The hourly average electricity generation of 30 MW WEC field (Figure 7, bottom) exhibits a different pattern. Wind turbine generation is typically higher during the night and lower during the day. This is especially apparent in Jul-Aug, when average wind output averages just over 5 MW (17\% of nameplate capacity) at $12 \mathrm{~h}$, and a peaks at $12 \mathrm{MW}$ at $21 \mathrm{~h}-22 \mathrm{~h}$. This is substantial variation considering it is an average over 120 days (two months for two years).

As Figure 7 suggests, the probability of Digby exporting electricity, is a function of both the season 312 and the time of day. This probability is more fully described in Figure 8. Both heating loads and 313 wind production increase during the winter, but the frequency of exporting power are more closely 314 tied to the variations in WEC power output, so in colder months, exports are more common. Over 315 the day, the negative correlation between load, which peaks during the day, and WEC output, which 316 peaks at night, mean that exports are always $20-25 \%$ more likely at night. 


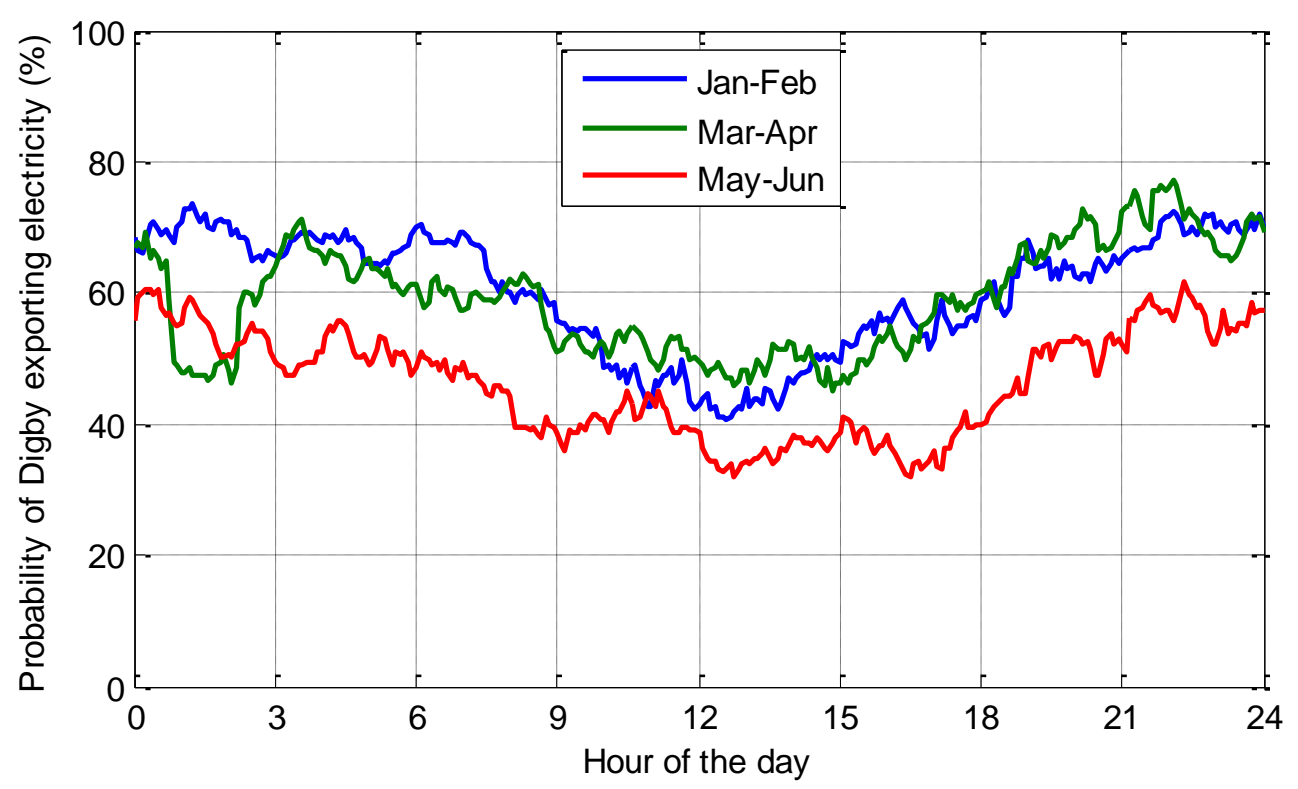

Hour of the day

Figure 8 Probability of Digby exporting electricity through the year.

319 Of course Figure 7 and Figure 8 represent general trends only, and at any given time of the year, the 320 WEC field might be producing nothing, or might be at its maximum output of $30+\mathrm{MW}$.

\section{$322 \quad 4.2$ Impact of EV charging on the electricity grid}

323 To illustrate the impacts of the three charging scenarios on the electricity grid we examine the first

324 week of June, 2014, as shown in Figure 9. During this period, Digby Load (red, right axis) was quite

325 low at 4-8 MW. The WEC field's generation (green, left axis) experienced both periods of low 326 generation (such as June 3) and high generation (June 6). Consequently, Transmission Line L-5533

327 (blue, left axis) experiences periods of net import (positive values, e.g. June 3), and net export 328 (negative values, e.g. June 6). The reader should note that export values approaching -26 MW are of 329 concern because of transmission constraints. 


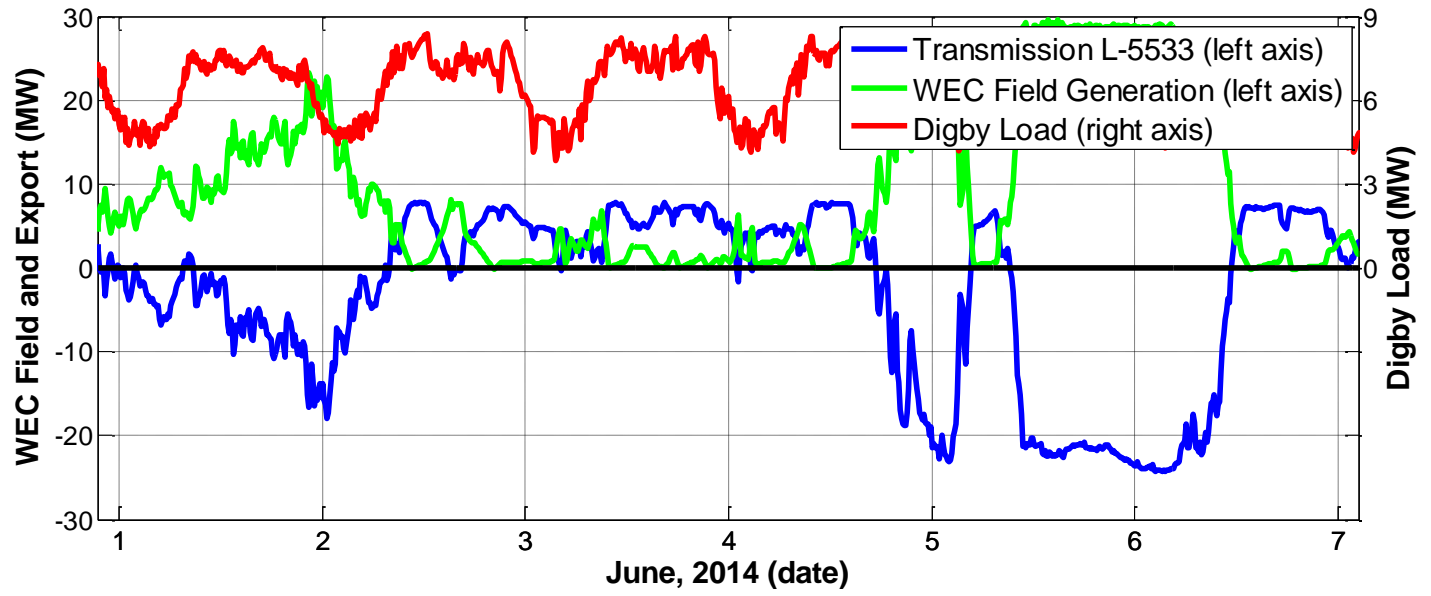

Figure 9 Electricity flows of transmission, generation, and load in Digby in June

In Figure 10, the fleet charging load profiles described in section 3.2 are plotted as a timeseries for the same week in June, 2014, along with the unmodified export power timeseries from Figure 9. Charging loads for convenience charging (yellow), TOD charging (cyan) and ideal smart charging (magenta) are read on the right axis, while unmodified export power on L-5533 (blue) is read on the left axis to clearly illustrate times of concern for export.

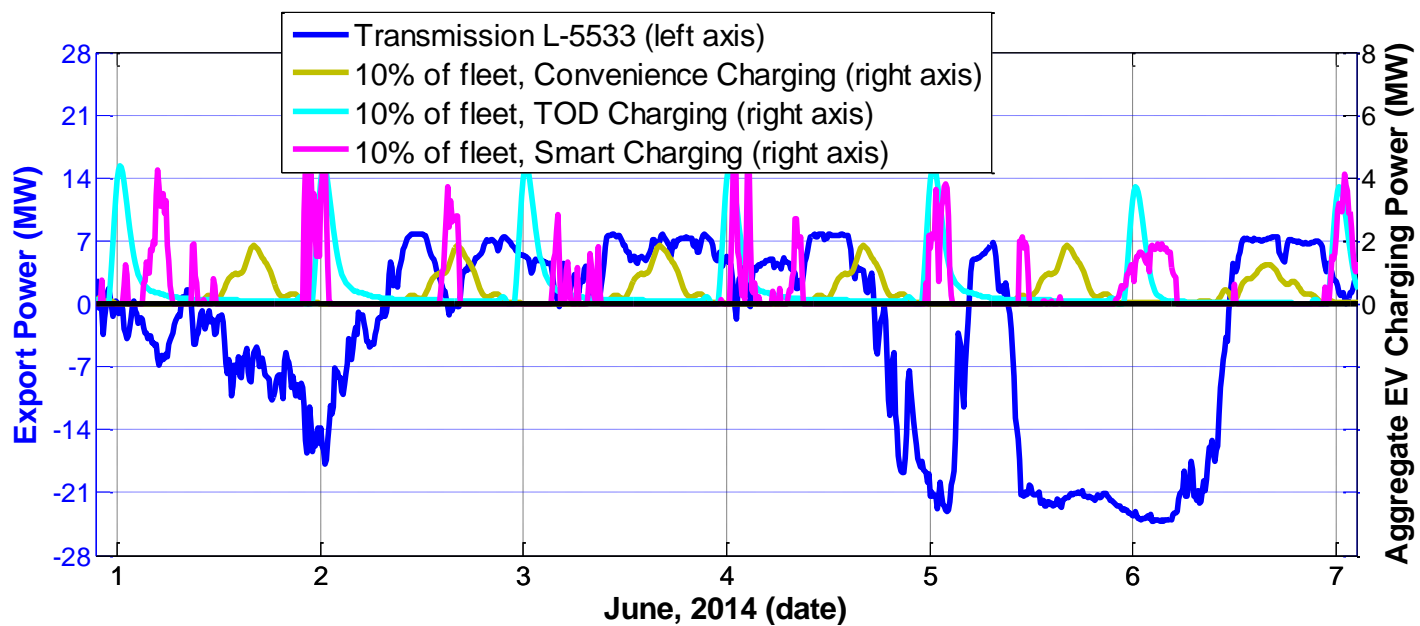

Figure 10 Electricity profiles of Convenience, TOD, and Smart charging compared with unmodified Transmission L-5533 in June

Figure 10 shows that convenience charging (yellow) adds about $2 \mathrm{MW}$ of load quite consistently, with the load somewhat normally distributed around $17 \mathrm{~h}$. This is the likely EV load scenario if no 
342 policy and/or tariff is put in place to encourage EVs to charge at specific times. The TOD charging 343 scenario (cyan) will add over $4 \mathrm{MW}$ of load (slightly less on weekends), which ramps up quickly 344 every night beginning at 23h. The smart charging scenario (magenta) can add as much as 6 MW of 345 load (10 kW per vehicle), coordinated precisely with each day's peak exports on L-5533. It is 346 evident that smart charging is highly variable in power, which will cause EVs to charge at various 347 rates when signalled. It is likely that EV owners would be willing to accept such signals given an 348 appropriate tariff or incentive, so long as they occurred overnight, thus having minimal impact on 349 vehicle readiness for travel.

350 Note that, when the assumed smart charging logic is applied to June 3 (a low wind day) it causes the 351 majority of the charging to occur in the morning when vehicles are likely in use. As previously 352 stated, this unrealistic behavior is not problematic for the overall results, because such conditions, 353 when local loads are not at their minimum, do not negatively impact the export transmission 354 infrastructure.

355 Examining the loads within the local distribution area, the three charging algorithms result in 356 significantly modified load profiles, shown in Figure 11 . This is an aggressive case (10\% adoption 357 rate) used to demonstrate the trends and impacts of EVs on the electricity grid. 


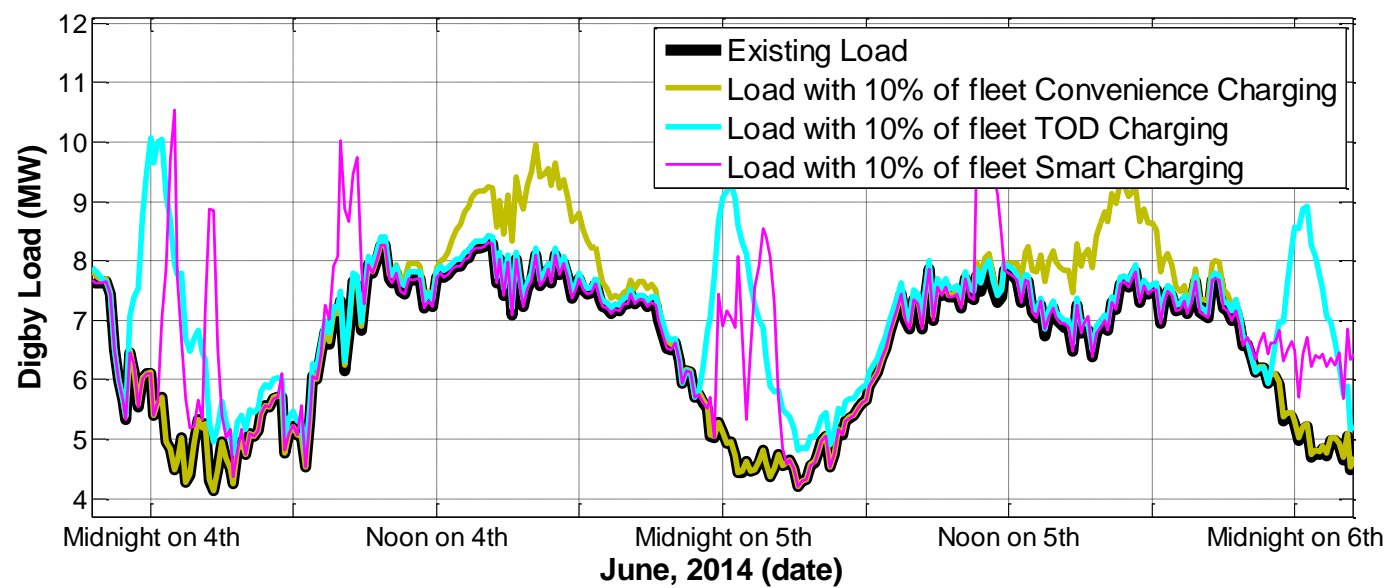

To determine how EVs might actually help provide load to address the grid constraint on

370 Transmission L-5533, their influence is directly applied to L-5533 power to create a modified load

371 time-series in Figure 12.

Figure 11 Time-series plot of electricity demand on the distribution lines out of Conway substation (77V) in the first week of June, 2014, given three possible EV charging scenarios being implemented by $10 \%$ of the vehicle fleet.

The size of these changes to the local load profile is striking. The magnitude of these responses is due to the relative scales of variations in local load and variations in wind output. Convenience charging (yellow) adds significantly to the evening loads, causing the daily load variability to increase from about $4 \mathrm{MW}$ (peak to trough) to 5 or $6 \mathrm{MW}$. TOD charging (cyan) adds load when they are low overnight, and it can be clearly identified that these start at $23 \mathrm{~h}$ with the drop in electricity cost. Load due to smart charging (magenta), is both inconsistent in timing, and abrupt, though Figure 11 shows that it does exhibit some correspondence to charging at times of low load. 


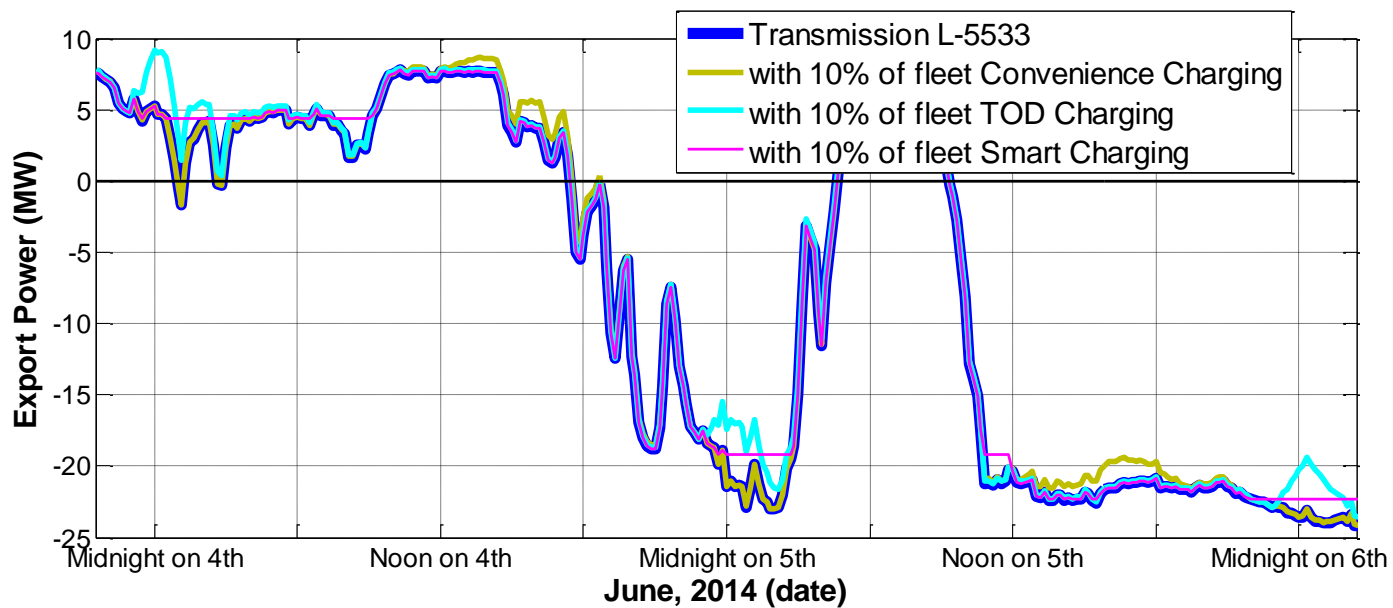

Figure 12 Time-series plot of electricity exports out of the Digby Neck area in the first week of June, 2014. The unmodified line power (black line), and line power resulting from three EV charging algorithms are shown (green, red, and cyan).

In Figure 12, it can be seen that convenience charging (yellow) does modify the L-5533 export. On June 4, it is not helpful, adding load when L-5533 was importing electricity already. On June 5 convenience charging happens to reduce relatively high exports, but not reduce the maximum exports of the day. TOD charging (cyan) also provides helpful load to modify L-5533. It reduced exports from -23 MW to -21 MW on June 5. It is also helpful on June 6. Smart charging (magenta) is seen in Figure 12 to cause highly controlled load variation on L-5533. This can be noted by the horizontal magenta lines delineating modified exports. On June 5 it reduces export from -23 MW to -19 MW. On June 6 it reduces export from -24 MW to -22 MW. These modification occur precisely when required, as this is an idealized implementation.

To more broadly quantify the effects of charging algorithm, each was applied to every day during the first half of 2014, the period for which detailed generation output and transmission system data were available. The original export power curve and the three resulting modified export power curves were then sorted into exceedance probability curves, which are presented in Figure 13 in linear (left) and logarithmic (right) plots. 

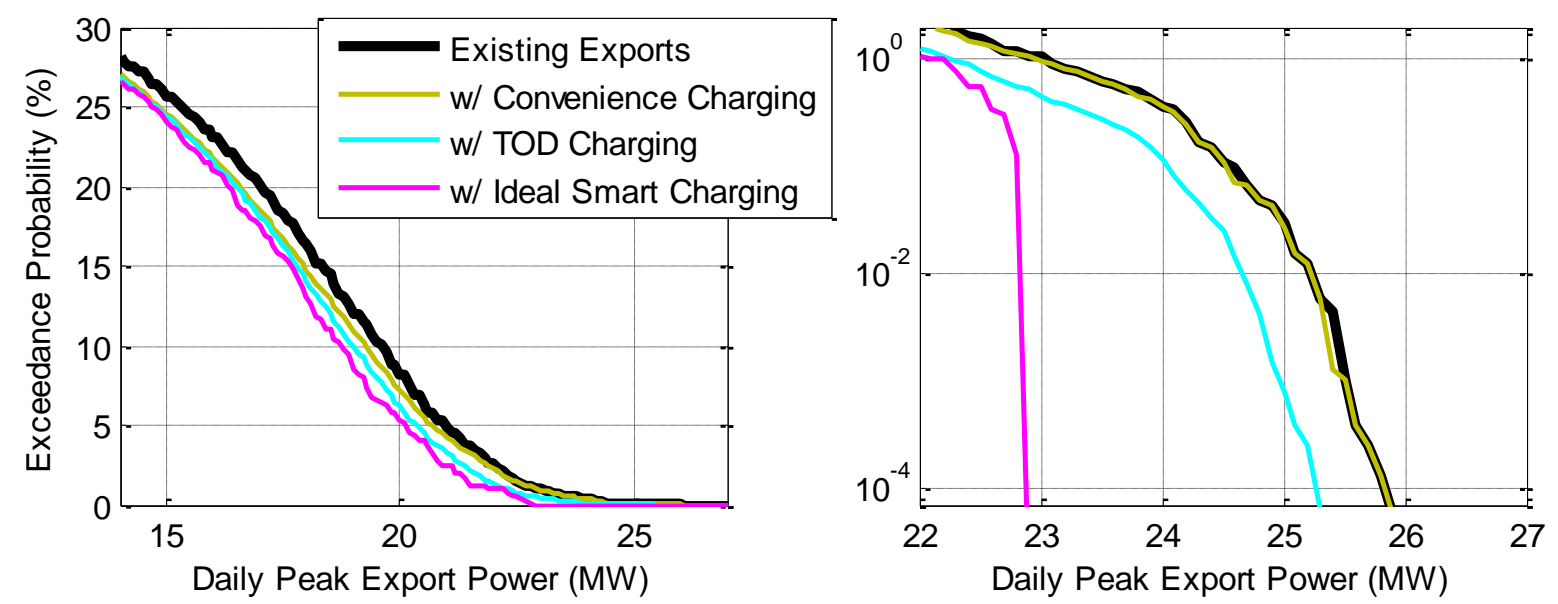

Figure 13 Line 5533 export power, exceedance probability, showing effects of three charging strategies applied to $10 \%$ of Digby vehicle fleet

393 The plot on the left of Figure 13 shows a broad range of export powers, and shows that all charging

394 algorithms reduce the frequency of exports somewhat compared to the existing export exceedance

395 probability (black line). However, export powers below about $23 \mathrm{MW}$ are of no concern since they

396 do not challenge the transmission capacity. In the right plot of Figure 13 the same data are shown in

397 greater detail, showing only exports between $22 \mathrm{MW}$ and $27 \mathrm{MW}$, which correspond to exceedance

398 probabilities of about $2 \%$ and less, and using a logarithmical distribution for the $y$-axis. From the

399 right plot of Figure 13, it is evident that convenience charging (yellow) has very little effect on the

400 most challenging export conditions. In contrast, TOD charging (cyan) reduces the worst exports by

401 about 0.6 MW, while Ideal Smart Charging (magenta) reduces the worst exports by $3 \mathrm{MW}$. 


\section{Discussion}

403 Because of the high probability that Digby will be a net exporter of renewable energy at any given

404 time (as shown in Figure 8), any EV charging in Digby will often be using locally generated 405 renewable electricity. Specifically, even using the convenience charging algorithm, about $49 \%$ of 406 EVs' energy would be from local WEC output. Using the TOD charging strategy, this fraction is 407 improved to 59\%, as charging events are pushed to the overnight hours when exports are more 408 likely (Figure 8). Using real-time control to find each day's period of peak export in the smart 409 charging algorithm means that $85 \%$ of the time EVs would charge from local WEC-sourced power.

410 While these fractions make EVs seem attractive for local consumers, it is worth noting that any

411 renewable electricity subtracted from the greater grid supply will require additional generation 412 elsewhere [21]. Thus the greater question is whether any charging strategies can facilitate adding 413 increased renewables to the grid, and whether such additional capacity can make up for the 414 additional energy demand to power the EV fleet. This question can be answered from the preceding analysis, as shown in Figure 14 and discussed below.

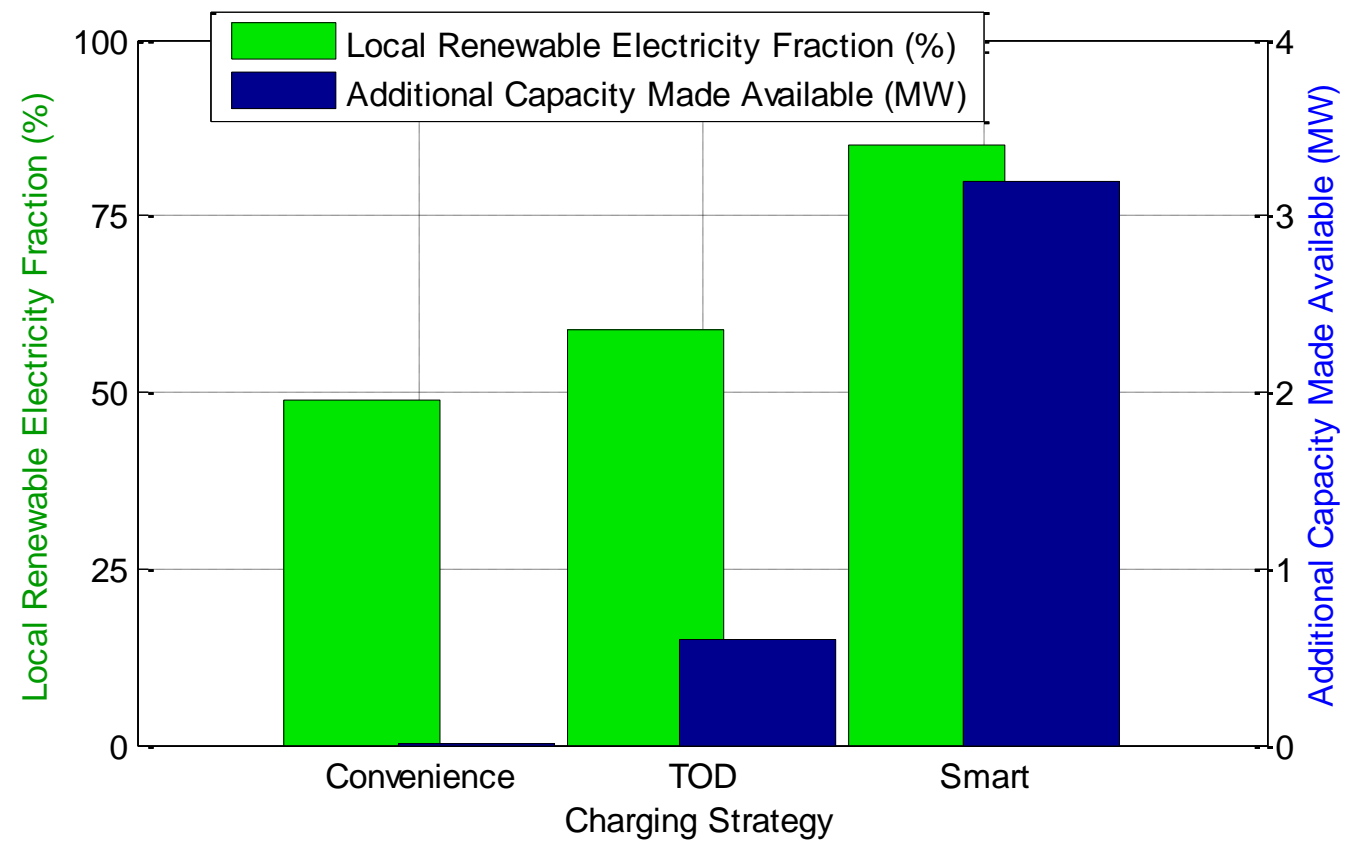


417 Figure 14 Synopsys of effects of charging strategies

418 The TOD charging strategy, implemented for 10\% of Digby's vehicle fleet, has been found to reduce 419 export peaks by about 0.6 MW. At the same time, the fleet consumes about $9 \mathrm{MWh}$ of electricity 420 each day. In order for 0.6 MW of additional capacity to provide the annual energy to power such a 421 fleet, it would need to have an annual capacity factor of about $62 \%$. Thus the answer to the question 422 of whether these additional cars can be powered from local renewable electricity is, unfortunately, 423 'no', unless an exceptionally good renewable resource, significantly better than the existing WEC 424 field, can be found.

425 The smart charging strategy, in contrast, could free up 3 MW of export capacity. An additional 426 renewable energy resource with an annual average capacity factor of just $12 \%$ would therefore 427 fully power the fleet of smart charging vehicles. As has been discussed, the load profile derived 428 from the smart charging scenario is idealized and could not fully be realized as it requires $100 \%$ 429 participation, arbitrarily high charging power per vehicle, and vehicles to be plugged in and ready 430 to accept charge precisely when needed. Determining exactly how such an idealized algorithm 431 would translate into a real world response is beyond the scope of this paper and would require an 432 extremely detailed knowledge about not only drive cycles but about vehicle owners' responses to 433 whatever incentive structure is put in place.

434 That being said, it seems reasonable to assume that the export attenuation resulting from real 435 world smart charging would be more effective at the stated goal of capping export power than the 436 convenience TOD response. If real world smart charging could attenuate export peaks by 1 MW 437 (compared to $3 \mathrm{MW}$ for ideal smart charging), and thereby free up $1 \mathrm{MW}$ of transmission capacity 438 to new generation, then that renewable resource would have to produce at $37 \%$ capacity factor to 439 produce all of the $9 \mathrm{MWh} /$ day needed by the vehicle fleet. Since the existing WEC produces at a 440 similar capacity factor, this is a very favorable finding. 


\section{Conclusions}

442 Digby is a region with abundant renewable energy resources, but has existing electricity grid 443 constraints. Digby has articulated an ambitious goal to alter its energy consumption and production

444 to better utilize locally produced renewable energy. Many such regions presently exist, and many 445 more will develop given the ubiquity of renewable energy generation policy. Electric vehicles can 446 support such policy as they reduce energy consumption for transportation, and can use locally 447 generated electricity from renewable resources, while acting as controllable loads. The objective of 448 this study was to determine the impact that EVs have on the electricity grid for various charging 449 control strategies, and if this is complementary to renewable energy generation.

Three charging strategies were evaluated for their effect on the interaction between renewable electricity generation and export transmission constraints. Convenience charging (not signalled)

452 will occur in absence of any policy or electricity tariff. This will add electricity load to Digby, but will 453 do so at existing peak load periods (17h) when export capacity is of no concern. The use of either 454 time of day charging (scheduled) or smart charging (signalled) will incent drivers to charge overnight, or when additional load is most needed to alleviate electricity grid constraints.

Scheduled and signaled charging strategies would increase the fraction of transportation energy sourced from local renewable electricity generators from an already high $49 \%$ for convenience charging, to 59\% for TOD charging, or $85 \%$ for smart charging. More importantly, these strategies were shown to be effective at addressing grid considerations: Using a $10 \%$ EV adoption rate, such charging algorithms could provide 0.6 to $3 \mathrm{MW}$ of additional transmission capacity. This could enable new renewable energy generation, such as a negligible cost WEC field control strategy upgrade, that on an annualized basis could provide all the energy needed to power the vehicle fleet. 


\section{Acknowledgements}

466 We thank Terry Thibodeau and Brittany Carroll of the Municipality of the District of Digby who 467 carried out many of the transportation surveys and provided contact information for key 468 transportation users. John Charleton and Stephen Ritcey at Nova Scotia Power kindly provided the 469 electricity system data and maps. We are grateful for the funding provided by the Nova Scotia 470 Department of Energy through the Nova Scotia Moves program, and to the Municipality of the 471 District of Digby.

472

473

474

475 References

476

477 [1] Statistics Canada, "Report on energy supply and demand in canada; 2011 preliminary," Statistics

478 Canada, Ottawa, Canada, Tech. Rep. 57-003-X, April 2013. 2013.

479 [2] Nova Scotia Department of Energy. (March 11, 2009). Nova Scotia Wind Atlas.

480 [3] R. H. Karsten, J. M. McMillan, M. J. Lickley and R. D. Haynes. Assessment of tidal current energy in 481 the minas passage, bay of fundy. Proceedings of the Institution of Mechanical Engineers Part A 482 Journal of Power and Energy 222(5), pp. 493-507. 2008. . DOI: 10.1243/09576509JPE555. 
483 [4] J. A. Carr, J. C. Balda and H. A. Mantooth, "A survey of systems to integrate distributed energy 484 resources and energy storage on the utility grid," in Energy 2030 Conference, 2008. ENERGY 2008. 485 IEEE, 2008, pp. 1-7.

486 [5] P. Denholm, E. Ela, B. Kirby and M. Milligan, "The role of energy storage with renewable 487 electricity generation," NREL, Tech. Rep. NREL/TP-6A2-47187, 2010.

488 [6] D. Connolly, H. Lund, B. V. Mathiesen, E. Pican and M. Leahy. The technical and economic 489 implications of integrating fluctuating renewable energy using energy storage. Renewable Energy 490 43(0), pp. 47-60. 2012.. DOI: 10.1016/j.renene.2011.11.003.

491 [7] J. Druitt and W. Früh, "Simulation of demand management and grid balancing with electric 492 vehicles, ISSN $\quad$ 0378-7753, http://dx.doi.org/10.1016/j.jpowsour.2012.05.033. 493 (http://www.sciencedirect.com/science/article/pii/S0378775312008907) Abstract: Keywords: " 494 Journal of Power Sources, vol. 216, pp. 104-116, 15 October 2012, 2012.

495 [8] W. Kempton and J. Tomić, "Vehicle-to-grid power implementation: From stabilizing the grid to 496 supporting large-scale renewable energy," J. Power Sources, vol. 144, pp. 280-294, 6/1, 2005.

497 [9] M. Metz and C. Doetsch, "Electric vehicles as flexible loads e A simulation approach using 498 empirical mobility data," Energy, vol. 48, pp. 369-374, December 2012, 2012.

499 [10] L. Zhang, T. Brown and G. S. Samuelsen, "Fuel reduction and electricity consumption impact of 500 different charging scenarios for plug-in hybrid electric vehicles," J. Power Sources, vol. 196, pp. 501 6559-6566, 1 Aug 2011, 2011.

502 [11] M. D. Galus and G. Andersson, "Demand management of grid connected plug-in hybrid electric 503 vehicles (PHEV)," in Energy 2030 Conference, 2008. ENERGY 2008. IEEE, 2008, pp. 1-8. 
504 [12] Shengnan Shao, Tianshu Zhang, M. Pipattanasomporn and S. Rahman. Impact of TOU rates on 505 distribution load shapes in a smart grid with PHEV penetration. Presented at Transmission and 506 Distribution Conference and Exposition, 2010 IEEE PES. 2010, . DOI: 10.1109/TDC.2010.5484336.

507 [13] P. Dvorak, "GE's WindBOOST increases energy production for 2,000th wind turbine," 508 Windpower Eng\&Dev, vol. 2013, June 20, 2013. 2013.

509 [14] GE Wind, "Turn up. tune up. wind PowerUp; making your machines brilliant," General Electric, 510 Tech. Rep. GEA30884, Oct. 2013. 2013.

511 [15] (2014). Registry of Motor Vehicles.

512 [16] W. Sierzchula, S. Bakker, K. Maat and B. van Wee, "The influence of financial incentives and 513 other socio-economic factors on electric vehicle adoption

514 ," Energy Policy, vol. 68, pp. 183-194, May 2014, 2014.

515 [17] N. S. Pearre. Location, Duration, and Power; how Americans' Driving Habits and Charging 516 Infrastructure Inform Vehicle-Grid Interactions 2013.

517 [18] J. Axsen and K. S. Kurani, "Anticipating plug-in hybrid vehicle energy impacts in California: 518 Constructing consumer-informed recharge profiles," Transportation Research Part D: Transport 519 and Environment, vol. 15, pp. 212-219, 6, 2010.

520 [19] L. S. N. Pearre, "High-resolution residential electricity consumption trends under fixed and 521 time-of-use rates," in ESim Building Simulation Conference, Ottawa, 2014, .

522 [20] W. Hennings, S. Mischinger and J. Linssen, "Utilization of excess wind power in electric 523 vehicles," Energy Policy, vol. 62, pp. 139-144, November 2013, 2013. 
524 [21] A. Pina, P. Baptista, C. Silva and P. Ferrão, "Energy reduction potential from the shift to electric 525 vehicles: The Flores island case study," Energy Policy, vol. 67, pp. 37-47, April, 2014, 2014.

526 